\title{
PSICO-ONCOLOGIA E AMPARO A PACIENTES COM CÂNCER: uma revisão de literatura
}

DOI: $10.22289 / 2446-922 X . V 6 N 1 A 17$

\author{
Pâmela Schultz Danzmann 1 \\ Ana Claudia Pinto da Silva \\ Janaina Pereira Pretto Carlesso
}

\section{RESUMO}

O câncer na sociedade em que vivemos, ainda está associado a morte. Assim, a morte e o processo de morrer são pouco discutidos e muitas vezes nem mencionados nos pequenos e grandes grupos. O psico-oncologista surge nesse panorama como uma forma de apoio e suporte, para paciente, família e profissionais que estão envolvidos diariamente com essas experiências, de modo a estarem expostos a perda qualidade de vida psíquica e física devido a todo desgaste. O objetivo principal deste estudo foi discutir o papel do psicólogo e da psicologia dentro do contexto da Psico-oncologia, de modo a ressaltar a importância na formação do profissional sobre essa temática que muitas vezes é pouco explorada ou discutida durante a formação acadêmica. $O$ estudo foi embasado por meio de uma pesquisa bibliográfica. A partir disso, discutiu-se questões emocionais, fragilidades e cotidiano do paciente, família e equipe de saúde, e principalmente como o psicólogo trabalha diante das questões que surgem no contexto da Psico-oncologia. Conclui-se destacando a importância do profissional como base de suporte para todas as pessoas que são envolvidas em sua área de atuação.

Palavras-chave: Assistência ao Paciente; Patologia; Psicologia em Saúde.

\section{PSYCHO-ONCOLOGY AND SUPPORT FOR CANCER PATIENTS: a literature review}

\section{ABSTRACT}

Cancer in our society is still associated with death. Thus, death and the process of dying are little discussed and often not mentioned in small and large groups. The psycho-oncologist appears in this panorama as a form of support, for patient, family and professionals who are involved daily with these experiences, in a way exposed to loss of quality of psychic and physical life due to all wear and tear. The main objective of this study was to discuss the role of psychologist and the psychology whitin in the context of psycho-oncology, in order to emphasize the importance of this subject, that is often little explored or discussion during academic formation. The study was based on bibliographic research. From this, it discussed emotional issues fragilities and daily life of the patient, family and health team, and mainly how the psychologist work in the face of issues that

\footnotetext{
${ }^{1}$ Endereço eletrônico de contato: pamelapsicologia10@gmail.com

Recebido em 06/04/2020. Aprovado pelo conselho editorial para publicação em 27/04/2020. Rev. Psicol Saúde e Debate. Jul., 2020:6(1): 244-255
} 
arise in the context of psycho-oncology. It concludes by highlighting the importance of the professional as a support base for all people who are involved in the your area of expertise.

Keywords: Patient Assistance; Pathology; Health Psychology.

\section{PSICONCOLOGÍA Y APOYO PARA PACIENTES CON CÁNCER: una revisión de la literatura}

\section{RESUMEN}

El cáncer en la sociedad en que vivimos todavía está asociado con la muerte. Por lo tanto, la muerte y el proceso de morir rara vez se discuten y muchas veces ni siquiera se mencionan en grupos pequeños y grandes. El psicooncólogo aparece en este panorama como una forma de apoyo para el paciente, la familia y los profesionales involucrados diariamente con estas experiencias, para estar expuesto a la pérdida de la calidad de vida psicológica y física debido a todo el desgaste. El objetivo principal de este estudio fue discutir el papel del psicólogo y la psicología en el contexto de la Psico-oncología, con el fin de enfatizar la importancia en la formación de profesionales sobre este tema, que muchas veces se explora o discute poco durante la formación académica. El estudio se basó en la investigación bibliográfica. A partir de esto, se discutieron los problemas emocionales, las debilidades y la vida cotidiana del paciente, la familia y el equipo de salud, y principalmente cómo trabaja el psicólogo frente a los problemas que surgen en el contexto de la Psico-oncología. Concluye destacando la importancia del profesional como base de apoyo para todas las personas involucradas en su área de especialización.

Palabras clave: Atención al Paciente;Patología; Psicología de la Salud.

\section{INTRODUÇÃO}

O câncer surge no decorrer da história como um dos grandes medos que assombram a sociedade e até hoje é responsável pelo mal-estar psíquico e físico causado no indivíduo, desconsiderando classe social, gênero ou idade. Sua origem ainda é desconhecida pela ciência e sabe-se que existem pelo menos mais de duzentos tipos de patologias diferenciadas (Landskron, 2008).

Além disso, aqui discute-se uma doença crônica, de tratamento extenso e doloroso e que leva uma parcela significativa da população a óbito. Diante disso, inúmeras áreas da saúde, incluindo a psicologia, estão à procura de métodos de tratamento e prevenção para o câncer (Malta et al., 2014).

Nesse contexto de adoecimento e busca de uma vida saudável a Psicologia foi aos poucos sendo inserida, destacando-se como uma das disciplinas mais antigas no universo acadêmico. Assim Medicina e Psicologia, que implicam na busca da saúde, seja ela física, mental ou ambas vem evoluindo com o passar dos séculos em suas práticas e na forma de trabalhar com o ser humano (Mosimann \& Lustosa, 2011).

Rev. Psicol Saúde e Debate. Jul., 2020:6(1): 244-255 
Outra importante consideração a ser feita é que o adoecimento do corpo produz também uma instabilidade na subjetividade do sujeito. Sendo assim, o psicólogo, diferentemente de outros profissionais, estará interessado no significado subjetivo que a doença tem para o paciente. Ajudando-o através de uma escuta qualificada, que possibilite um espaço para a execução da palavra, e o auxiliará na elaboração simbólica (Cantarelli, 2009).

Muitas vezes, as pessoas que recebem o diagnóstico de câncer, resolvem se apegar em algo que sustente essa dor. A espiritualidade e a religiosidade acabam se tornando fundamentais para alguns pacientes, principalmente por serem consideradas como referência de descoberta para sua cura (Freire, Vasconcelos, Silva, \& Lima Oliveira, 2017).

Nesse panorama da pessoa com câncer surge o trabalho do Psico-oncologista, que conforme Hart (2008), atua na prevenção, no recebimento do diagnóstico, no tratamento e na finalização do tratamento, seja ela a cura ou a morte. Além disso, identifica variáveis psicossociais e ambientais para que o paciente enfrente a doença.

A partir de tais colocações, o objetivo principal desse estudo foi a discussão do papel do psicólogo e da Psicologia dentro do contexto da Psico-oncologia, de modo a ressaltar a importância na formação do profissional sobre a temática, que muitas vezes acaba por ser pouco investida ou discutida durante a graduação. Ademais, a pesquisa trata de dar ênfase tanto para os aspectos emocionais que envolvem o paciente, sua família e amigos, bem como toda a equipe médica envolvida nos cuidados.

Salienta-se também a importância do psicólogo que está diretamente envolvido em diversos contextos. Tanto no âmbito de quem necessita do cuidado, no caso o paciente, como também no âmbito daqueles que promovem o cuidado, seja a família ou a equipe de saúde.

\section{MÉTODO}

O estudo caracteriza-se como uma pesquisa bibliográfica de caráter qualitativo. Segundo o autor Gil (2009) conceitua esse tipo de pesquisa como sendo realizada a partir de livros, artigos científicos e base de dados eletrônicos. Além disso, uma das grandes vantagens é o acesso e uma gama de informações, que o pesquisador não poderia acessar diretamente.

A pesquisa sobre a temática ocorreu no primeiro semestre de 2020 , através das plataformas Google Scholar, Pepsic e Scielo. Os descritores utilizados na pesquisa foram: "Psicooncologia"; "Atuação do Psicólogo"; "Família"; e "Equipe médica". Foram selecionados artigos, monografias, resumos que falavam sobre o tema, correspondentes aos últimos anos, portanto são referentes do período de 2010 a 2020.

Também se utilizou de um livro físico que abordava questões relativas ao câncer. O livro é intitulado "Câncer, uma abordagem psicológica". A construção desse escrito, deve-se a AAPECAN 
(A associação de apoio da pessoa com câncer), que tem a finalidade de transmitir conhecimento e apoio psicológico a pessoa com câncer e seus familiares.

\section{PSICO-ONCOLOGIA: conceito, atuação e intervenções}

Antes de entendermos o âmbito daqueles que cuidam e daqueles que são cuidados, é importante conhecer um pouco mais sobre a função do psico-oncologista e de onde essa profissão deriva. Desse modo, o profissional que atua nessa área é responsável por promover qualidade de vida a todos indivíduos que se envolvem diretamente ou indiretamente no contexto da pessoa com câncer. No entanto, a Psico-oncologia é um uma vertente da Psicologia da Saúde e a inserção desse profissional muitas vezes se dá de forma interdisciplinar, onde buscam por novas maneiras das pessoas lidarem com o sofrimento advindo da patologia (Fonseca \& Castro, 2016).

Historicamente, é visto que a Psico-oncologia surge com a finalidade de dar suporte e apoio para o paciente diagnosticado com câncer. Os sintomas de angústia, depressão e ansiedades provocados pela doença, não estavam sendo resolvidos apenas com a medicação. A Psicooncologia com uma proposta diferenciada, onde estende o olhar para pacientes, familiares e equipe médica, trazendo uma possibilidade de compreensão do adoecer e considerando fatores biopsicossociais (Campos, 2010).

Ademais, é preciso considerar que a vida é constantemente delineada por processos de saúde e doença. Sendo assim, é importante a consideração de aspectos fundamentais como a subjetividade, aspectos sociais, religiosos, econômicos e políticos. A coleta e a considerações dessas informações reforça ainda mais a importância da atuação do psico-oncologista juntamente com os demais profissionais da saúde. Principalmente, ao fazer o acompanhamento de estatísticas que comprovam um aumento cada vez mais significativo de pessoas com câncer (Alves, Viana, \& Souza, 2018).

Outra consideração importante a ser feita é a discussão do processo interventivo realizado pelo psicólogo no contexto da pessoa com câncer. Destaca-se aqui que a literatura encontrada aborda as intervenções grupais como formas interventivas eficientes. Mas, o Hospital de Câncer de Barretos, trabalha com um modelo interventivo diferenciado, que seria um Programa de Assistência Domiciliar, para aqueles pacientes que devido às particularidades e limitações ficam impossibilitados de chegar até a instituição. $O$ atendimento é realizado semanalmente e a equipe de saúde atua de forma interdisciplinar, ela se desloca até a residência do paciente para atendê-lo (Shavanino et al., 2013).

Partindo dessa perspectiva, é possível perceber que a Psico-oncologia ainda é recente e pouco discutida, entretanto já houve um crescimento e evolução da mesma no Brasil, segundo os Rev. Psicol Saúde e Debate. Jul., 2020:6(1): 244-255 
estudo de Oliveira e Mello Andrade (2019), em decorrência do aumento das demandas em suporte e acolhimento psicológico em serviços que atendem pacientes oncológicos e seus familiares. Desse modo, estudos apontam um aumento significativo de formações e capacitações de psicólogos nessa área de atuação (Alves et al., 2016).

\subsection{Paciente e cuidador}

Ao adentrarmos no campo das pessoas que estão envolvidas com o câncer, entre elas paciente, sua família e a equipe médica, inúmeras considerações devem ser feitas. Entre elas, a fase terminal do indivíduo que está acometido com a doença, que de acordo com Silva Ferreira e Cavalcante (2017), é considerado o pior momento para o cuidador dessa pessoa. Pois, é nesse instante que o desgaste físico, emocional e financeiro chega ao seu ápice. O cuidador, segundo definição das mesmas autoras, será aquela pessoa que está diretamente comprometido a todo tipo de cuidado para com o paciente. O mesmo pode ser remunerado ou não. Além disso, suas atividades estão relacionadas aos cuidados relativos à higiene e saúde.

A Psicologia entra nesse panorama, diferentemente do passado, onde as pessoas á relacionavam diretamente com a loucura. Atualmente o profissional psicólogo conquistou novos espaços, dentre eles a inserção frente ao suporte e ao apoio no campo das doenças físicas. Segundo Hart (2008), o trabalho do profissional tanto a nível do paciente quanto de seus cuidadores, é através do auxílio para minimizar sentimentos de desesperança, abandono, isolamento, diminuir a ansiedade, bem como esclarecer dúvidas sobre tratamento e possibilidade de cura.

Mesmo depois da Psicologia conquistar novos espaços, deve-se considerar, que muitas pessoas ainda não distinguem de forma clara o psicólogo dos demais profissionais da saúde. Isso pode ser explicado devido a profissão ser muito recente quando comparada com as outras dentro da área de saúde (Pires et al., 2019). A Psico-oncologia surgiu no Brasil, inspirada em campos internacionais, nos anos 80 e assim criou-se centros de atendimentos psicossociais a fim de divulgar conhecimentos acerca do câncer e treinar os profissionais para o atendimento (Landskron, 2008).

Conforme Silva Ferreira e Cavalcante (2017), reportam uma questão de extrema importância que a Psicologia como qualquer profissão da área na saúde pode pecar, que seria a centralização do cuidado. Hoje ainda, o foco dos cuidados está na maioria das vezes relacionado ao paciente com câncer, e o cuidador fica a margem do acolhimento psicológico. Tem-se a falsa ideia que o cuidador pode auto cuidar-se, ademais é desconsiderado que esse indivíduo também necessita apoio e atenção. 
Ao retratar o cuidador e a família como um contexto único, deve-se considerar que a partir do momento em que o sujeito adoece, pode existir uma mudança radical na rotina. De acordo com Klug (2018), além das dúvidas e dores da família diante do adoecimento de um de seus membros, existe uma mudança na dinâmica familiar e uma reorganização cotidiana. Assim Psicologia, Medicina e as outras áreas da saúde podem atuar em conjunto estabelecendo um cenário de assistência a essa família e de consideração com suas necessidades. Segundo Costa e Ambrósio (2019), ainda no que se refere a mudanças na rotina familiar, o grupo muitas vezes se adapta a momentos incertos como internações, preocupações financeiras, tratamentos agressivos e o próprio âmbito hospital. Tudo isso pode afetar a qualidade de vida desses indivíduos.

\subsection{Psico-oncologia infantil}

A gravidez é caracterizada por momentos de idealização do filho. Dessa forma a mãe passa a imaginar o filho esperado e é natural que quando a criança nasce, ocorra um confronto do bebê real com o bebê imaginado (Ferreira, Jesus Costa, \& Couto, 2018). Ao levarmos em consideração o panorama da criança com câncer, essa colocação do autor tem a mesma validade, visto que se idealiza uma criança saudável e muitas vezes a ela pode nascer com câncer ou mesmo depois de um tempo desenvolvê-lo.

Na maioria das vezes quem enfrenta o diagnóstico infantil de câncer é a mãe. Assim, deixa de lado todos os outros papéis que assumia para ficar em função do cuidado com o filho doente. Deixa em algumas situações os outros filhos, trabalho, marido ou companheiro(a). Isso tudo pode implicar em conflitos com os familiares ou até mesmo sérias discussões. A Psicologia surge nesse contexto como uma forma de auxílio e apoio tanto para a criança quanto para a mãe e toda a família. Assim busca-se um ponto de equilíbrio para que a mãe cuide do filho doente e reestruture sua vida pessoal (Ferreira, 2008).

No sistema familiar quando um indivíduo do grupo adoece, existem incertezas e aspectos emocionais que atingem toda conjuntura familiar. A partir desse momento que é vivenciado com extrema angústia, existe uma busca excessiva por uma melhor qualidade de vida para todos envolvidos. Quando esse adoecimento se refere a uma criança com câncer essa realidade é ainda mais triste e angustiante, pois ocorre uma associação do adoecimento com a morte, o que implica na inversão do ciclo natural de vida. A Psicologia tem um papel fundamental neste âmbito, visto que, atua minimizando os conflitos psicológicos que surgem nos familiares e garantido que a equipe de saúde oferecerá um trabalho humanizado a família (Costa \& Ambrósio, 2019). De acordo com as autoras anteriormente citadas, a busca por uma qualidade de vida saudável é fundamental para superar aspectos psicológicos envolvidos na doença, como o medo e a angústia que surgem com a confirmação da doença.

Rev. Psicol Saúde e Debate. Jul., 2020:6(1): 244-255 
Assim, Ferreira (2008) discute a importância do papel da escola como uma ponte de interação com outras pessoas. A autora salienta em seu estudo o quanto é fundamental para a criança adoentada que não perca o contato com seus colegas e amigos por medo ou vergonha da doença. Por outro lado, a escola também deve atuar positivamente ao acolher a criança e respeitar suas necessidades e limitações na sua volta.

Além disso, quando a criança é instruída a desenvolver suas habilidades sociais são desencadeados inúmeros benefícios psicológicos e físicos, principalmente a superação do isolamento. Ao utilizar o seu tempo para a prática de atividades agradáveis, a criança poderá se distrair, superar a fadiga por meio da leitura, música, desenhos (Cely-Aranda, Aritizabal, \& Capafons, 2013).

Mais uma vez ressalta-se a importância da atenção que se deve ter com os cuidadores, pois no caso da criança, conforme Costa e Ambrósio (2019), ela é uma parte da totalidade desse grupo, formada pelas vivências e construções da família e volta-se a refletir o contexto grupal. Cuidar da criança, ouvi-la, remete nas mesmas ações para com seus familiares. Todo esse atendimento especializado atrelado a atuação do psicólogo, possibilita uma melhor qualidade de vida da família e benefícios frente ao quadro de câncer infantil.

\subsection{Psico-oncologia e morte}

A morte começa a se manifestar quando nascemos. Aceitar a morte e a perda são experiências difíceis, pois nesse momento se rompe o vínculo com alguém. Perder uma pessoa de quem se gosta, é um processo doloroso, que desorganiza e assusta o sujeito, causando-lhe sofrimento. No entanto, é necessário a aceitação e superação desse momento para dar continuidade a vida. As pessoas que estão em processo de luto, na maioria das vezes preferem o isolamento, resultado dos medos e sofrimento da perda (Hart, 2008).

No momento em que a doença avança, de maneira a não ter mais nada a fazer, os cuidados paliativos são fundamentais para a garantia de qualidade de vida nesses últimos momentos. Tudo isso é garantido pela equipe de saúde responsável, que tenta sempre buscar o melhor para seu paciente (Torres, 2018).

O ser humano dever ser considerado como um todo, corpo e mente, para o seu funcionamento. Pois a apresentação dos sintomas não é só orgânica, mas também psicológica.Os sentimentos que surgem diante da vulnerabilidade que a doença provoca, devem ser considerados. Isso porque as pessoas que estão psicologicamente fragilizadas, estão mais suscetíveis as doenças. Tudo isso decorre devido a imunização baixar, justamente pela delimitação emocional do indivíduo( Hart, 2008). 
Ainda no que se refere aos profissionais atuantes, o psicólogo nesse contexto é fundamental. Pois o diagnóstico de morte é recebido com extrema angústia e sofrimento. $O$ psicólogo realizará a escuta do paciente e da família e tentará trazer novas percepções sobre a doença e morte. Além disso, servirá de suporte durante todo tempo que for necessário, e pode utilizar de técnicas como os grupos terapêuticos com a finalidade de minimizar o sofrimento. Se achar necessário, o profissional da Psicologia, além de tudo que já foi citado, pode sugerir mudanças no comportamento do paciente frente a morte (Fonseca \& Castro, 2016).

Nesse caso sempre é respeitado, escutado e acolhido a pessoa em sofrimento físico e psíquico (Torres, 2018). Conforma a mesma autora, os cuidados paliativos são fundamentais nesses últimos momentos. Algumas vezes, o paciente pode ter uma melhora significativa, sentese acolhido e confiante e isso também se deve ao profissional da Psicologia. Essa confiança é tamanha que família e paciente criam um vínculo que transpassa a doença, no sentido de poderem conversar sobre tantas outras coisas além da doença ou morte.

Para Aguiar, Gomes, Ulrich, e Mantuani (2019), os cuidados paliativos têm o significado de aceitar a morte como um processo. Para isso, o profissional além de sustentar uma boa escuta, deve fazer o possível para que esse paciente tenha uma boa qualidade de morte, tornando o mesmo o protagonista de seu processo de morrer. Além disso, o trabalho deve ser interdisciplinar, de modo que envolva toda a equipe e a boa comunicação seja assegurada.

Outra questão a ser considerada, segundo Hart (2008) é que quando o familiar vive em função dos cuidados de um doente por muito tempo, e esse vem a falecer pode surgir um sentimento de vazio. O preenchimento desse vazio é um processo difícil, visto que, por muito tempo o familiar abdicou de sua vida para o cuidado. Nesses casos, o processo de luto pode ser dificultoso. No caso do luto mal elaborado, o sujeito pode sentir-se ansioso, triste, ter surtos psicóticos, idealização suicida, entre outras problemáticas. Assim, mais uma vez o trabalho do psicólogo deve ser ressaltado, principalmente para que o familiar consiga integrar seus objetos internos, receba atenção e o tratamento necessário para enfrentar a situação.

Uma das ferramentas utilizadas pelo psicólogo para lidar com a doença e a morte é terapia grupal. Pode ser utilizado para pacientes e familiares que passam por momentos difíceis. Em conformidade com Bermudez (2008), existem diversos benefícios aos participantes, como a identificação de pessoas que passam pela mesma situação, construção de vínculos afetivos, troca de experiências emocionais, abrange conflitos intrapsíquicos que em conjunto vão sendo superados, entre outros.

De acordo com Ferreira (2015), relata que tanto os encontros grupais quanto os atendimentos individuais trazem inúmeros benefícios para o paciente com câncer. Dessa forma, expectativas, oportunidades e possibilidades passam a ser repensadas no contexto em que se encontram, e deixa-se de lado as dificuldades do tratamento mudando o foco que até então era 
somente a doença. Além disso, discute a importância de uma formação profissional que possa ceder um espaço não só para o entendimento, como também para a atuação no contexto da pessoa com câncer.

Nos últimos anos, diretrizes estão sendo estabelecidas para uma prática clínica melhorada dentro do panorama da pessoa com câncer. As propostas são feitas pela National Comprehensive Cancer Network (NCCN) e pelo Institute of Medicine (IOM). Dentro desse contexto, uma recomendação importante que precisa ser mencionada é a avaliação das necessidades psicossociais e o sofrimento emocional dos pacientes, para que sejam urgentemente encaminhados para os recursos adequados (Galindo-Vázquez \& Costas Muniz, 2019). A grande dificuldade nesse sentido é que os programas de Psico-oncologia possuem limitações e são insuficientes para o atendimento de todos os pacientes (Vaccaro et al., 2019).

Por fim, e não menos importante, cabe salientar que o psico-oncologista deve atentar-se também para a equipe de saúde que dedica seu tempo aos cuidados dos pacientes com câncer. É muito como, que com o passar do tempo, cercados muitas vezes de uma rotina adversa e desafiadora, tais profissionais venham a desenvolver uma alta carga tensional. Nesses casos, desgaste físico, mental e profissional bem como a fadiga podem sinalizar que as coisas não andam bem (Aguiar et al., 2019).

\section{CONSIDERAÇÕES FINAIS}

O estudo aponta que ao se adentrar no campo universitário da Psicologia, percebe-se a partir das leituras, que muitas universidades não discutem profundamente questões relativas à morte e ao morrer. Assim, muitas vezes os acadêmicos têm um conhecimento raso sobre a Psicologia da Saúde e principalmente sobre a Psico-oncologia. A não ser que busquem tal conhecimento de maneira autodidata ou por meio da experiência de estágios na área. Ademais, a psico-oncologia, algumas vezes é pouco reconhecida ou mesmo distorcida até mesmo dentro do campo da saúde.

Ainda hoje, existe uma forte associação do câncer com a morte, que é intensificado devido a doença não ter uma cura. Dessa forma, quando o paciente recebe o diagnóstico, logo entra em desespero, imaginado uma morte próxima. Além disso, ocorrem diversas mudanças na vida do individuo que foi diagnosticado e da sua família. Podem surgir limitações e dificuldades devido a introdução de uma rotina nova e muitas vezes cansativa. O papel do psicólogo nesse sentido, seria ajudar o paciente a se adaptar com essas mudanças físicas, psicológicas e algumas vezes sociais que a doença implica.

Outro ponto importante a destacar é que doença e saúde são processos que fazem parte da vida. Nesse sentido é preciso pensar na subjetividade do sujeito que foi diagnosticado com 
câncer, considerar condições econômicas, sociais, políticas que envolvem o indivíduo, em outras palavras considerar o paciente como um todo.

O psicólogo que atua diretamente com o paciente acometido com câncer, não trabalha sozinho nunca, ao contrário do que se espera muitas vezes de um atendimento clínico individual. O profissional atenta-se a questões relativas à subjetividade ou perda dela em um contexto hospitalar, onde o paciente e sua família passam por um processo adaptativo. Assim, estão sujeitos a normas, regras e um cotidiano totalmente diferente daquele de costume. Como se fosse pouco, para ajudar a desequilibrar ainda mais a saúde psíquica desses sujeitos, o câncer tem uma forte associação com a morte, todavia só se pode esperar a cura ou a morte.

O câncer não considera as condições econômicas, sexo ou idade. Dessa forma o psicólogo que deseja atuar nessa área deve ter em mente que desde crianças indefesas e frágeis idosos podem ser diagnosticados. Deve-se preparar também para ser o suporte de famílias que vivem em função de seus entes queridos e dão tudo de si, descuidando de sua saúde psíquica e física em nome desse cuidado. Ao mesmo tempo não se deve esquecer que existe uma equipe técnica na área de saúde que também necessita de um olhar psicológico, pois muitas vezes podem lutar exaustivamente pelo outro, e essa exaustão trazer sérias mazelas.

A partir da análise realizada torna-se evidente, portanto, que o profissional serve como uma base para paciente, família e a equipe de saúde. Seu papel é de extrema importância desde a prevenção até o final do tratamento, e a sua atuação traz benefícios para todos envolvidos nesse processo. Ao pensar nisso, mesmo que haja uma literatura vasta da temática, se comparar com outros temas discutidos na Psicologia, deixa-se aqui como sugestão de algo que foi pouco extraído na literatura. Sugere-se então que pesquisadores da área desenvolvam mais pesquisas e construções de estudos de campo que discutam e apresentam as intervenções e todos os benefícios que estão sujeitos às pessoas envolvidas no trabalho do psico-oncologista, bem como as dificuldades em atuar num âmbito que fica entre a vida e a morte.

\section{REFERÊNCIAS}

Aguiar, M. A. F., Gomes, P. A., Ulrich, R. A., \& Mantuani, S. B. de. (2019) Psico-oncologia: caminhos do cuidado. São Paulo: Summus.

Alves, G.S, Viana, J. A., \& de Souza, M. F. S. (2018). Psico-oncologia: uma aliada no tratamento de câncer. Pretextos-Revista da Graduação em Psicologia da PUC Minas, 3(5), 520-537. http://periodicos.pucminas.br/index.php/pretextos/article/view/15992

Bermudez, C.S.(2008). Psicoterapia Grupal na oncologia: psicooncologia com metodologia psicodramática (p.78-90). In C. F. B. Hart et al., (Orgs), Câncer: uma abordagem psicológica. Editora AGE LTDA, Porto Alegre.

Campos, E.M. P. A psico-oncologia. (2010). Bol. Acad. Paulista de Psicologia, 30( 79), 440-449. https://www.redalyc.org/pdf/946/94615412015.pdf

Rev. Psicol Saúde e Debate. Jul., 2020:6(1): 244-255 
Cantarelli, A. P. S. (2009). Novas abordagens da atuação do psicólogo no contexto hospitalar. Revista da SBPH, 12(2), 137-147. http://pepsic.bvsalud.org/pdf/rsbph/v12n2/v12n2a11.pdf

Cely- Aranda C. J., Aristizabal, C. P. D., \& Capafons, A. (2013). Psicooncología pediátrica. Diversitas: $\quad$ Perspectivas en Psicología, 289-304. http://www.scielo.org.co/scielo.php?script=sci arttext\&pid=S1794-99982013000200005

Costa, I. C. S. D., \& Ambrozio, L. C. (2019). Câncer infantil: Acompanhamento psicológico para a qualidade de vida familiar. Anais do I e do II seminário de produção científica do curso de Psicologia da Unievangélica. Anápolis, Brasil. http://45.4.96.19/bitstream/aee/1132/1/C\%c3\%82NCER\%20INFANTILACOMPANHAMENTO\%20PSICOLOGICO....pdf

Ferreira, R. (2008) Psicooncologia Pediátrica (p-32-42). In C. F. B. Hart et al., (Orgs), Câncer: uma abordagem psicológica. Editora AGE LTDA, Porto Alegre.

Ferreira, C. B. (2015). A construção do cuidado em psico-oncologia em um projeto de extensão $\begin{array}{llll}\text { universitária. } & \text { Psicologia em } & \text { 521-527. }\end{array}$ http://www.periodicos.uem.br/ojs/index.php/PsicolEstud/article/view/29554/pdf

Ferreira, I. C., de Jesus Costa, J., \& do Couto, D. P. (2018). Implicações do diagnóstico de autismo para a vivência da maternidade. Pretextos-Revista da Graduação em Psicologia da PUC Minas, 3(5), 431-448. http://periodicos.pucminas.br/index.php/pretextos/article/view/15936

Fonseca, R., \& Castro, M. M. (2016). A importância da atuação do psicólogo junto a pacientes com câncer: uma abordagem Psico-Oncológica. Psicologia e Saúde em debate, 2(Ed. Esp. 1), 54-72. https://doi.org/10.22289/2446-922X.V2EEA5

Freire, M. E. M., Vasconcelos, M. F., Da Silva, T. N., \& de Lima Oliveira, K. (2017). Assistência espiritual e religiosa a pacientes com câncer no contexto hospitalar. Revista de Pesquisa: Cuidado é fundamental online, 9(2), 356-362. http://dx.doi.org/10.9789/21755361.rpcfo.v9.4906

Galindo-Valquez, O., \& Carlos-Muniz, R. (2019) Evidence-Based Psycho- oncology: A priority in Mental Health. Salud Mental, 42(3) 101-102. https://www.medigraphic.com/pdfs/salmen/sam2019/sam193a.pdf

Gil. A. C. (2009). Métodos e técnicas de pesquisa social. 6. ed. São Paulo: Atlas.

Godoy, A. S. (1995). Pesquisa qualitativa: tipos fundamentais. Revista de Administração de empresas, 35(3), 20-29. http://www.scielo.br/pdf/rae/v35n3/a04v35n3.pdf

Hart, C. F. B. (2008). Perdas e processo de Luto. (p.153-166). In C. F. B. Hart et al., (Orgs), Câncer: uma abordagem psicológica. Editora AGE LTDA, Porto Alegre.

Klug, J. D. (2019). Psico-oncologia: à escuta do sujeito frente ao adoecimento por câncer. Monografia. Trabalho de Conclusão do Curso de Psicologia. Universidade Regional do Noroeste do Estado do Rio Grande do Sul - UNIJUÍ, ljuí, Brasil. http://bibliodigital.unijui.edu.br:8080/xmlui/handle/123456789/5803

Landskron, L. M. F. (2008). Psicooncologia: as descobertas sobre o câncer ao longo da vida (p.1131). In C. F. B. Hart et al., (Orgs), Câncer: uma abordagem psicológica. Editora AGE LTDA, Porto Alegre.

Rev. Psicol Saúde e Debate. Jul., 2020:6(1): 244-255 
Malta, D. C., Moura, L. D., Prado, R. R. D., Escalante, J. C., Schmidt, M. I., \& Duncan, B. B. (2014). Mortalidade por doenças crônicas não transmissíveis no Brasil e suas regiões, 2000 a 2011. Epidemiologia e Serviços de Saúde, 23, 599-608. https://doi.org/10.5123/S1679$\underline{49742014000400002}$

Mosimann, L. T. N. Q., \& Lustosa, M. A. (2011). A Psicologia hospitalar e o hospital. Revista da SBPH, 14(1), 200-232. http://pepsic.bvsalud.org/scielo.php?script=sci arttext\&pid=S1516$\underline{08582011000100012}$

Oliveira, E., \& de Mello Andrade, M. C. (2019). A Psico-oncologia e seus diversos desdobramentos. Revista Mosaico, 10(1). https://doi.org/10.21727/rm.v10i1.1685

Pires, R. A., Souza, I. C. D. S., Pereira, J. M., Lima, R. S. G. S., Quintana, R., \& Souza, M. C. D. (2019). A Psicologia no contexto de produção do cuidado segundo a percepção de pessoas com doença oncológica. Revista da SBPH, 22(1), 328-348. http://pepsic.bvsalud.org/scielo.php?script=sci arttext\&pid=S1516-08582019000100017

Scannavino, C. S. S., Sorato, D. B., Lima, M. P., Franco, A. H. J., Martins, M. P., M., Júnior, J. C., Bueno, P. R. T., Rezende, F. F., \& Valério, N. I. (2013). Psico-Oncologia: atuação do psicólogo no Hospital de Câncer de Barretos. Psicologia USP, 24(1), 35-53. http://www.scielo.br/scielo.php?script=sci arttext\&pid=S0103-65642013000100003

Silva Ferreira, G. M., \& Cavalcante, D. M. (2017). A saúde psíquica dos cuidadores familiares de pacientes acometidos com câncer. CIÊNCIA AMAZÔNIDA, 1(2). http://www.periodicos.ulbra.br/index.php/amazonida/article/view/3027/2561

Torres, A. A. (2018). Cuidados paliativos: A atuação do psicólogo com pacientes com câncer sem expectativa de vida. Pretextos-Revista da Graduação em Psicologia da PUC Minas, 3(6), 361 376. http://periodicos.pucminas.br/index.php/pretextos/article/view/15930

Vaccaro, L. Shaw, J., Sethy, S., Kirsten, L., Beatty, L., Mitchell, G., \& Turner, J. (2019). Barriers and facilitators to community-based psycho-oncology services: A qualitative study of health professionals' attitudes to the feasibility and acceptability of a shared care model. PsychoOncology. 28(9):1862-1870. https://www.ncbi.nlm.nih.gov/pubmed/31257660 